\title{
CONTRAST ENHANCEMENT FOR COLOR IMAGES USING AN ADJUSTABLE CONTRAST STRETCHING TECHNIQUE
}

\author{
Zohair Al-Ameen
}

Department of Computer Science, College of Computer Science and Mathematics, University of Mosul, Nineveh, Iraq, qizohair@gmail.com, http://www.uomosul.edu.iq/

Paper history:

Received 17 January 2018

Received in revised form 08 April 2018

Accepted 17 April 2018

Available online 30 June 2018
Keywords:

adjustable contrast stretching;

color image;

contrast enhancement;

image processing;

low-contrast.

\begin{abstract}
With the growing demand for high-quality color images, efficient yet low-complexity methods are increasingly needed for better visualization. Unfortunately, the low-contrast is one prevalent effect that degrades color images due to various unavoidable limitations. Hence, a new adjustable contrast stretching technique is proposed in this article to improve the contrast of color images. The processing scheme of the proposed technique is relatively simple. It starts by converting the input color image to grayscale. Then, it automatically computes two contrast tuning parameters depending on the pre-determined grayscale image. Finally, it improves the contrast of the degraded color image using an amended version of an existing contrast stretching technique. Accordingly, its input is a color image and a contrast adjustment parameter $\delta$, while its output is a contrast-adjusted color image. The proposed technique is tested by conducting intensive experiments on real-degraded images, and it is compared with four well-known contrast enhancement techniques. In addition, the proposed and comparative techniques are evaluated based on three eminent no-reference image quality assessment metrics. From the performance analysis of the achieved experiments and comparisons, the proposed technique provided satisfying performances and outperformed the comparative techniques in terms of recorded accuracy and perceived quality.
\end{abstract}

Copyright (C) Research Institute for Intelligent Computer Systems, 2018. All rights reserved.

\section{INTRODUCTION}

With the growing demand for high-quality color images, efficient yet low-complexity methods are increasingly needed for better visualization. However, the existence of the low-contrast effect can lead to a reduction in the ability of the observer to analyze and interpret important information in digital images [5]. Many reasons can lead to the occurrence of this effect, including low-light environment, faulty imaging device, lack of operator skills, poor environmental conditions and so forth [6]. Such effect can be dealt with using various types of contrast enhancement techniques.

The foremost aim of such techniques is to improve the perceived quality and reveal the latent information of a given degraded image so that it becomes visually better for further analysis and interpretation [7]. Currently, most of the available enhancement techniques involve either high- complexity or histogram based operations. In various situations, histogram modification-based techniques can cause inconvenient contrast enhancement, which eventually gives the processed image an unnatural appearance with visual artifacts [8].

Other than histogram equalization [18], there exist various contrast enhancement concepts including, sigmoid function [19], homomorphic filtering [20], log and power-law transformations [21], contrast stretching [22], retinex theory [23], fuzzy set [24] artificial bee colony [25], and so forth. Despite that, there still exist wide scopes for developing low-complexity techniques that are not histogram-based and can produce satisfactory results. Such techniques can be highly beneficial, especially for systems that have limited resources.

Hence, the proposed technique has been developed with the intention of providing an efficient yet fast process for contrast enhancement of 
color images. The processing scheme of the proposed technique is relatively simple. It starts by converting the input color image to grayscale. Then, it automatically computes two contrast tuning parameters depending on the pre-determined grayscale image. Finally, it improves the contrast of the degraded color image using an amended version of an existing contrast stretching technique. Accordingly, its input is a color image and a contrast adjustment parameter $\delta$, while its output is a contrast-adjusted color image.

To achieve this study, three factors have been taken into consideration. The first factor is the utilized image dataset, in which only real-degraded color images are utilized for experimental and comparison purposes. The reasons behind that are many types of imaging devices that have different hardware and software settings are available, as well as, each captured image has different environmental conditions. Thus, using only real-degraded images can allow a good opportunity to assess the true processing ability of the proposed technique.

The second factor is the employed comparison techniques, in which four advanced techniques of scaling in the discrete cosine transform domain by a quadratic mapping function with blocking artifact removal (TW-CES-BLK) [9], non-parametric modified histogram equalization (NMHE) [10], exposure-based sub image histogram equalization (ESIHE) [11], and recursive exposure based sub image histogram equalization (RESIHE) [12] were used for comparison purposes. The third factor is the utilized image quality assessment (IQA) metrics.

Generally, there exist three types of IQA metrics, namely full-reference, reduced-reference and noreference [13]. Accordingly, no-reference metrics require only one image as input, while the fullreference metrics usually require two images as input, which is the degraded or processed image and a reference image. In between these two extremes lie the reduced-reference metrics, which require certain information about the reference image, but not the actual reference image itself, in addition to the degraded or processed image [14].

Since only real-degraded images are utilized, only no-reference IQA metrics were used to measure the quality of the obtained results. Accordingly, three eminent metrics of average local contrast (ALC) [15], colorfulness (CFN) [16], and measure of enhancement (EME) [17] were used for such purpose. The organization of this article is as follows. After introducing an adequate overview in Section 1, the proposed technique is fully explained in Section 2. Afterwards, the experimental results with their related discussions are presented in Section 3. Finally, a succinct conclusion is given in Section 4.

\section{ADJUSTABLE CONTRAST STRETCHING TECHNIQUE}

The main idea of the proposed technique is based on the concept of linear contrast stretching (LCS), which has been typically used in various image processing applications [1]. There exist many techniques that can achieve LCS, in which their intricacy varies depending on the utilized concept. One of such LCS techniques can be expressed as [2]:

$$
\hat{W}=\Upsilon(W+\Gamma),
$$

where, $W$ is a given grayscale image; $\Upsilon$ and $\Gamma$ are contrast tuning parameters; $\hat{W}$ is a contrast-enhanced image. Although this technique can adjust the dynamic range of an image, it does not always yield satisfactory results, especially for images with major spatial variation in contrast [1]. Moreover, the values of parameters $\Upsilon$ and $\Gamma$ are not determined easily, since their determination procedures vary from one application to another.

In addition, this technique has been used basically for grayscale images, but occasionally for color images. Hence, a new adjustable contrast stretching technique is introduced which exploits a modified version of the above LCS technique. In brief, the parameters $\Upsilon$ and $\Gamma$ are computed automatically in the proposed technique based on the spatial and statistical information of an estimated grayscale version of the input image.

Furthermore, an additional parameter is added to control the amount of contrast enhancement in the resulting image. Finally, the input image is processed using an amended version of Eq. (1). Going into details, the proposed technique starts by computing the relative luminance, which represents the grayscale version of the input color image. The relative luminance can be determined as follows [3]:

$$
E=0.2126 I_{R}+0.7152 I_{G}+0.0722 I_{B},
$$

where, $E$ is the obtained relative luminance image; $I_{R}, I_{G}, I_{B}$ are the red, green, blue channels of the input color image $I_{R G B}$. For the sake of contrast improvement, the two contrast tuning parameters are determined automatically to be used later in the enhancement process. The used tuning parameters $\Gamma$ and $\Upsilon$ are computed as follows:

$$
\begin{gathered}
\Gamma=\delta\left[\frac{1}{n-1} \sum_{i=1}^{n}\left(E_{i}-\bar{E}\right)^{2}\right], \\
\Upsilon=1+(\Lambda \Gamma),
\end{gathered}
$$

where, $\delta$ is a parameter that controls the amount of contrast enhancement and it should fulfill $\delta>0$, in which a higher value leads to further contrast 
enhancement; $E_{i}$ is a vector version of image $E ; \bar{E}$ is the mean of $E_{i} ; n$ is the number of elements at the longest dimension of $E_{i} ; \Lambda$ is a regularization parameter that helps to avoid the increase of unnecessary whiteness, in which natural whiteness is produced when $\Lambda=1.4$. This value was determined from intensive experiments on various real lowcontrast color images. Parameter $\Gamma$ is computed by multiplying $\delta$ by an unbiased sample variance of $E$ [4], while parameter $\Upsilon$ is determined automatically based on the values of $\Lambda$ and $\Gamma$.

The reason behind that is to reduce the number of calculations involved in the proposed technique. Lastly, the contrast of the input color image is processed using the pre-determined tuning parameters with an amended version of Eq. (1). Improving the contrast of image $I_{R G B}$ is achieved using the following equations:

$$
T=\Upsilon\left(I_{R G B}-\Gamma\right),
$$

where, $T$ is the final output of the proposed technique. In Eq. (1), the reason behind changing the $(+)$ operator to $(-)$ is to provide better contrast for the resulting image. To end with, the subsequent pseudo-code is given to provide a precise description regarding the execution specifics of the proposed technique.

\begin{tabular}{l}
\hline The pseudo-code of the proposed technique. \\
\hline Input: low-contrast color image $I$ \\
Input: parameter $\delta$ \\
Set: parameter $\Lambda$ (default=1.4) \\
Compute the relative luminance using Eq. (2) \\
Compute parameters $\Gamma$ and $\Upsilon$ using Eq. $(3,4)$ \\
Process the contrast of image $I$ using Eq. $(5)$ \\
Output: contrast-improved color image $T$ \\
\hline
\end{tabular}

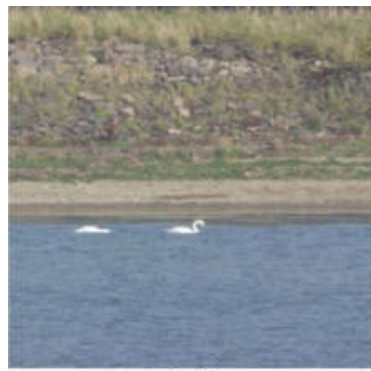

(a1)

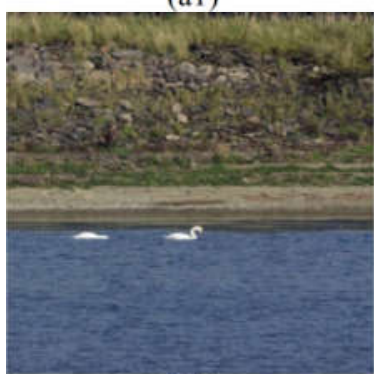

(b1)

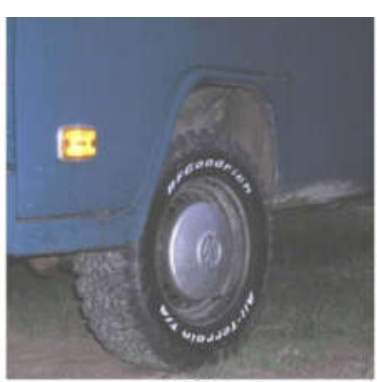

(a2)

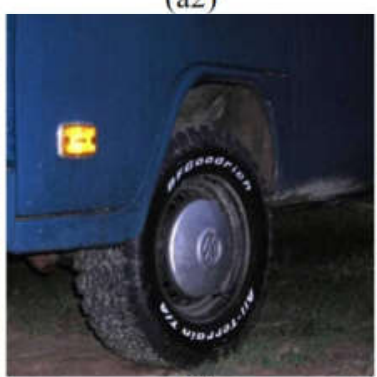

(b2)

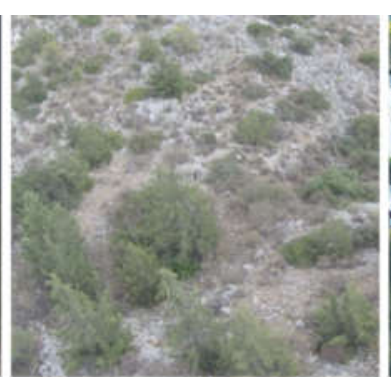

(a3)

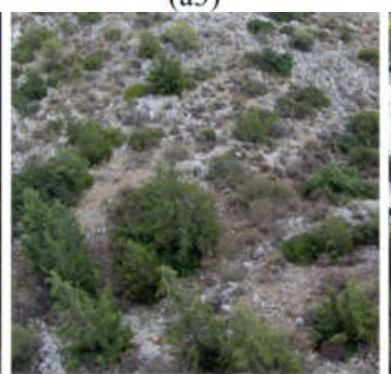

(b3)

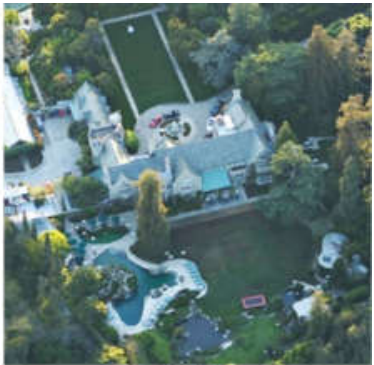

(a4)

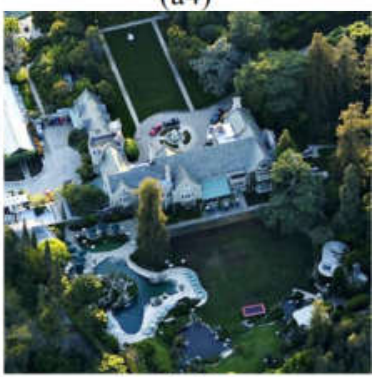

(b4)

Figure 1 - Applying the proposed technique to various real low-contrast color images. (a1-a4) are real-degraded images; (b1-b4) are images processed by the proposed technique with $\delta$ equal to 50,35, 20 and 6 , respectively. 


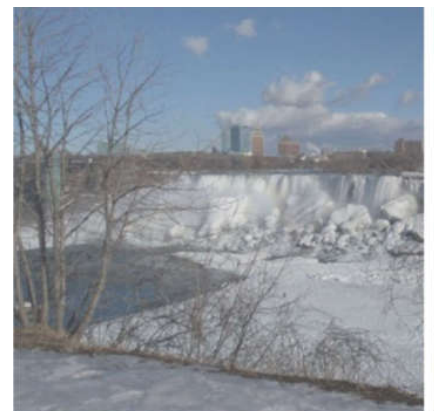

(a1)

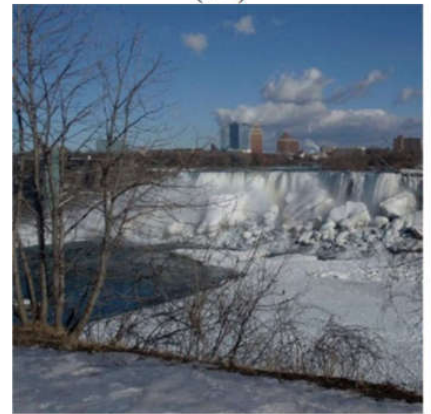

(b1)

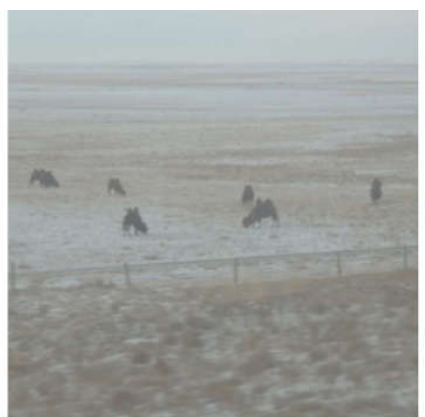

(a2)

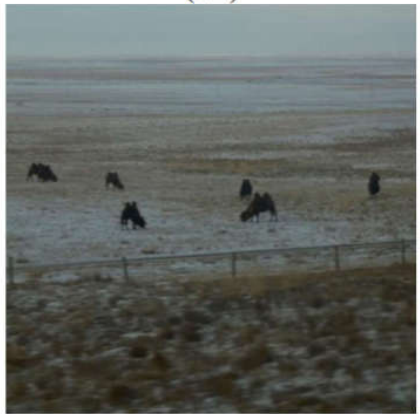

(b2)

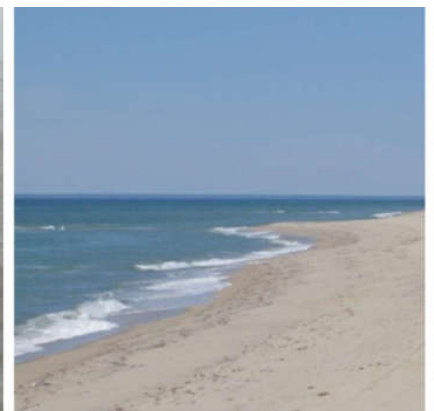

(a3)

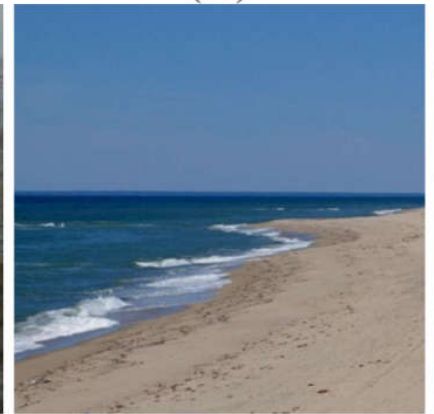

(b3)

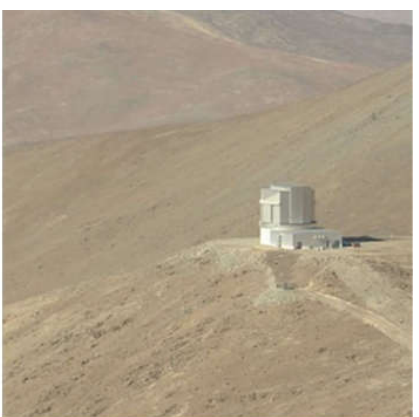

(a4)

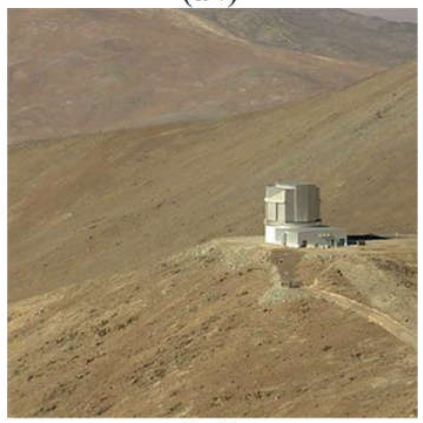

(b4)

Figure 2 - Applying the proposed technique to various real low-contrast color images. (a1-a4) are real-degraded images; (b1-b4) are images processed by the proposed technique with $\delta$ equal to $25,43,35$ and 90 , respectively.

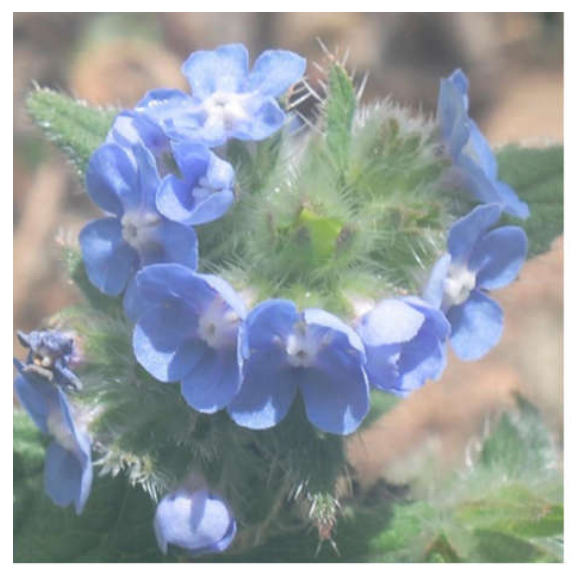

(a)

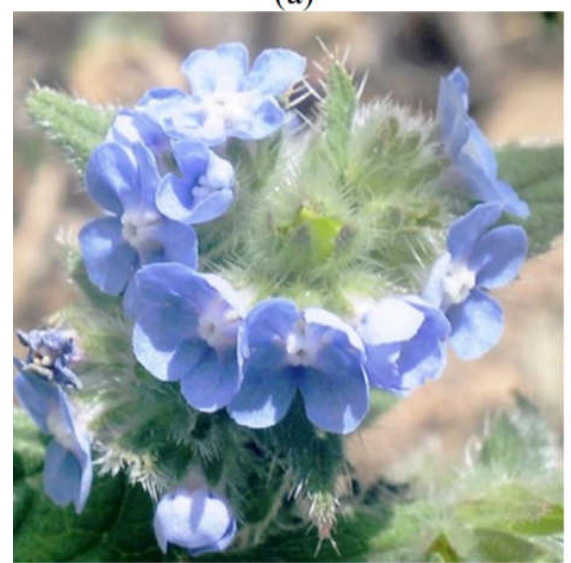

(d)

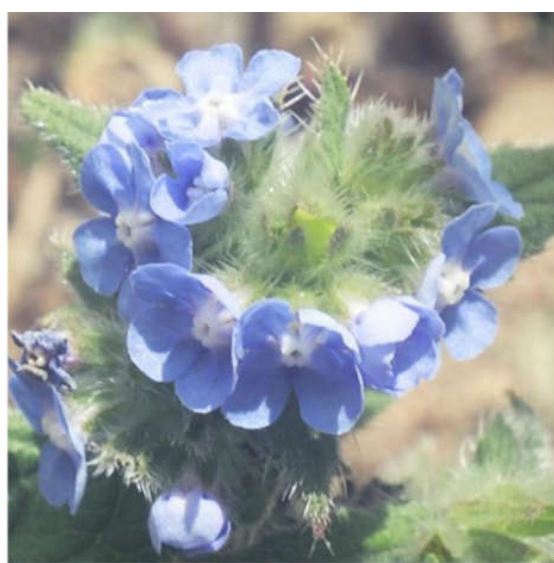

(b)

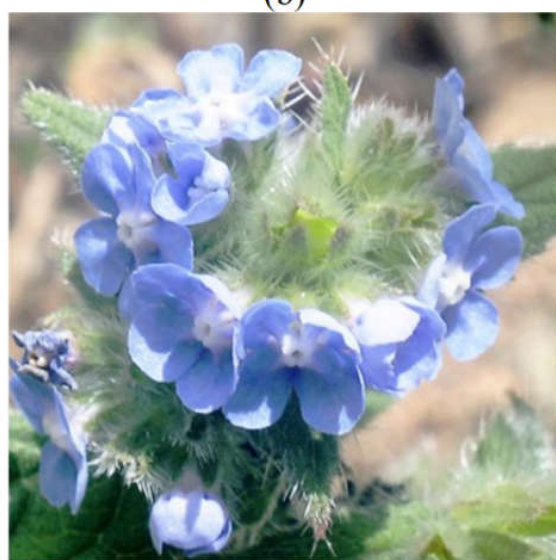

(e)

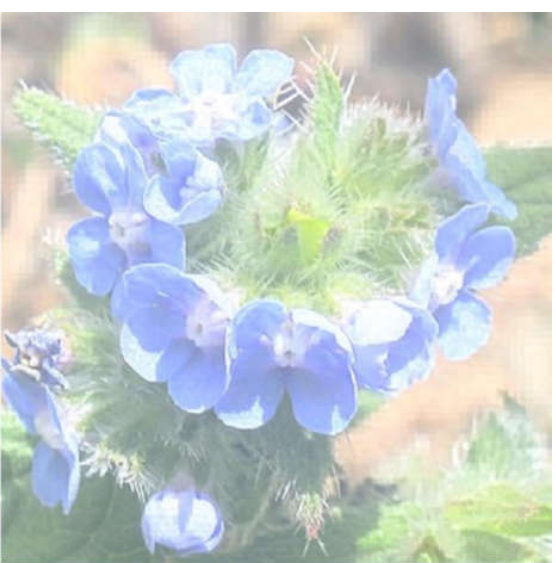

(c)

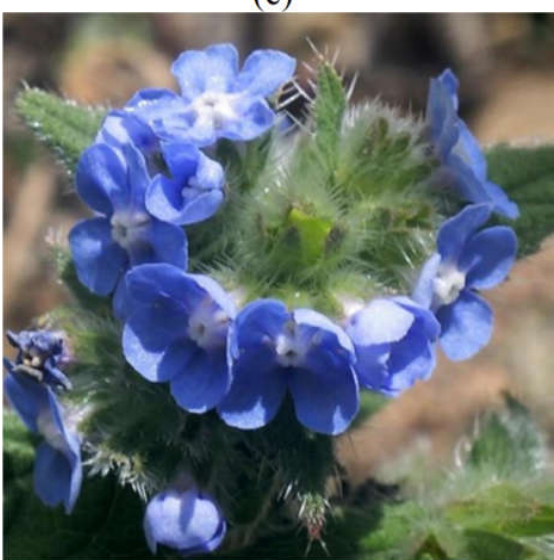

(f)

Figure 3 - Comparing the proposed technique with various renowned contrast enhancement techniques. (a) real degraded color image; images (b-f) are processed by: (b) NMHE; (c) TW-CES-BLK; (d) ESIHE; (e) RESIHE;

(f) proposed technique with $\delta=15$. 


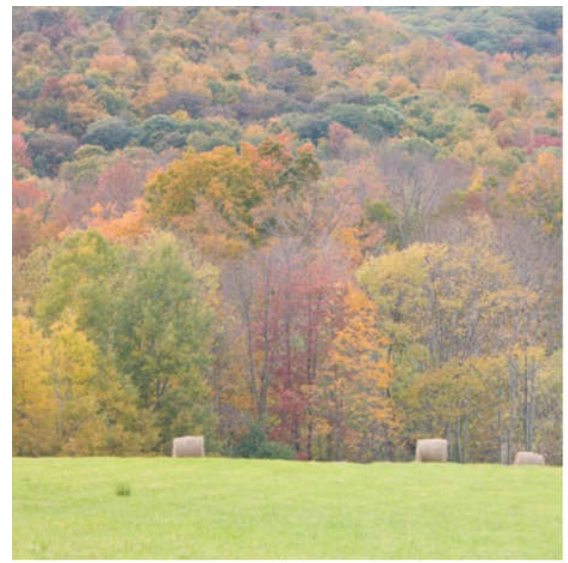

(a)

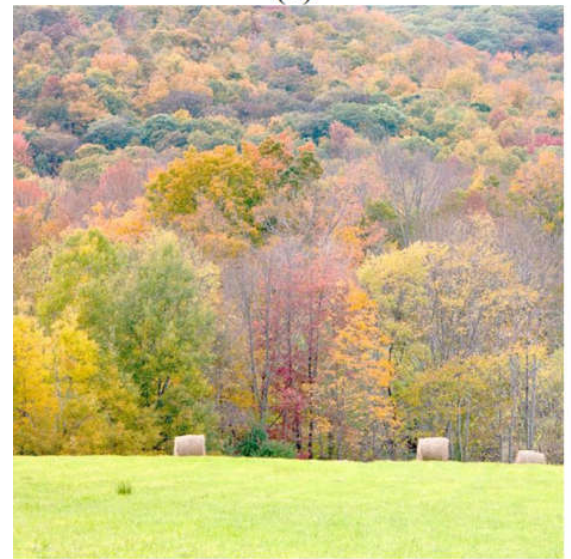

(d)

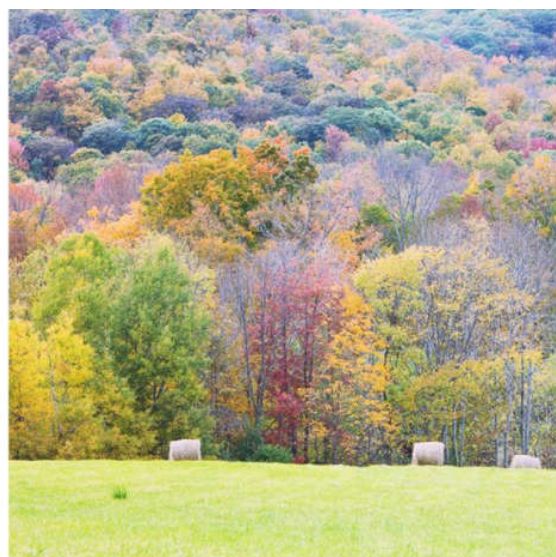

(b)

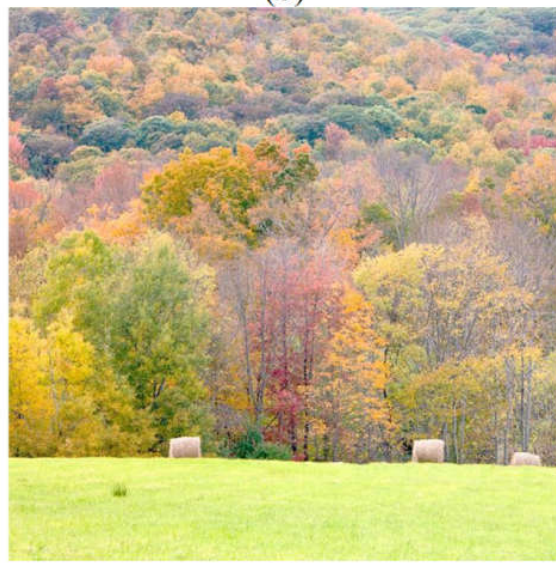

(e)

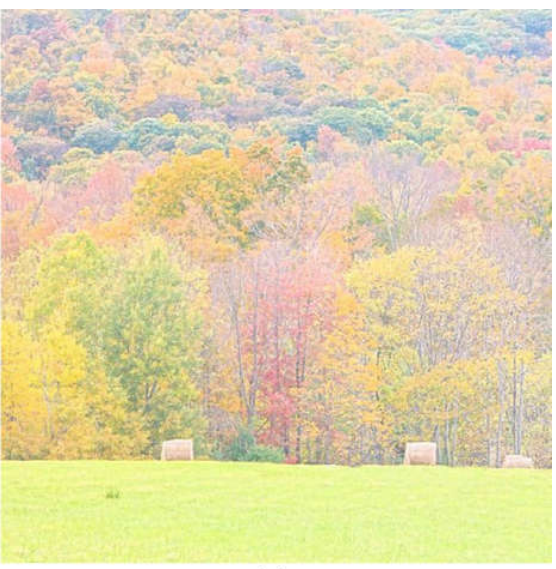

(c)

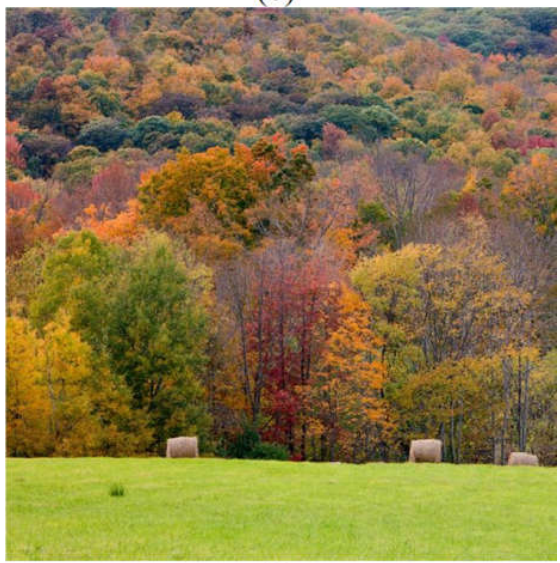

(f)

Figure 4 - Comparing the proposed technique with various renowned contrast enhancement techniques. (a) realdegraded color image; images (b-f) are processed by: (b) NMHE; (c) TW-CES-BLK; (d) ESIHE; (e) RESIHE; (f) proposed technique with $\delta=25$.

Table 1: The scored accuracies of the achieved comparisons.

\begin{tabular}{|l|l|c|c|c|}
\hline Techniques & Figures & CFN & EME & ALC \\
\hline \multirow{3}{*}{ Degraded } & Fig. 3 & 38.9599 & 4.6232 & 0.2934 \\
\cline { 2 - 5 } & Fig. 4 & 49.8266 & 9.0852 & 1.0891 \\
\cline { 2 - 5 } & Average & 44.3933 & 6.8542 & 0.69125 \\
\hline \multirow{3}{*}{ NMHE } & Fig. 3 & 42.7032 & 5.5907 & 0.4389 \\
\cline { 2 - 5 } & Fig. 4 & 61.1276 & 18.2470 & 2.6753 \\
\cline { 2 - 5 } Average & 51.9154 & 11.9189 & 1.5571 \\
\hline \multirow{3}{*}{ BL-CES- } & Fig. 3 & 41.1283 & 3.8004 & 0.2632 \\
\cline { 2 - 5 } & Fig. 4 & 54.0562 & 7.8269 & 1.1264 \\
\cline { 2 - 5 } & Average & 47.5923 & 5.81365 & 0.6948 \\
\hline \multirow{3}{*}{ RIHE } & Fig. 3 & 48.0844 & 11.7941 & 0.6651 \\
\cline { 2 - 5 } & Fig. 4 & 62.7667 & 19.1130 & 1.8474 \\
\cline { 2 - 5 } & Average & 55.4256 & 15.4536 & 1.25625 \\
\hline \multirow{3}{*}{$\begin{array}{l}\text { Proposed } \\
\text { Technique }\end{array}$} & Fig. 3 & 49.9667 & 12.8859 & 0.7234 \\
\cline { 2 - 5 } & Fig. 4 & 65.2783 & 21.0232 & 2.1082 \\
\cline { 2 - 5 } & Average & 57.6225 & 16.9546 & 1.4158 \\
\cline { 2 - 5 } & Fig. 3 & $\mathbf{5 4 . 2 9 0 5}$ & $\mathbf{1 4 . 3 6 2 9}$ & $\mathbf{0 . 8 7 3 6}$ \\
\cline { 2 - 5 } & Average & $\mathbf{6 6 . 7 5 8 3}$ & $\mathbf{5 2 . 7 5 8 2}$ & $\mathbf{3 . 9 6 7 9}$ \\
\hline
\end{tabular}

Note: The bold values indicate the best-achieved results

From the obtained experimental results in Fig. 1 and Fig. 2, it can be seen the proposed technique delivered satisfactory results, as it improved the drably appearance of the degraded images and provided adequate contrast, acceptable colors with no brightness amplification or visible flaws. In addition, the colors came out conspicuously leading the resulting images to have a more natural appearance. Thus, the processed images became clearer and more suitable for real-life usage.

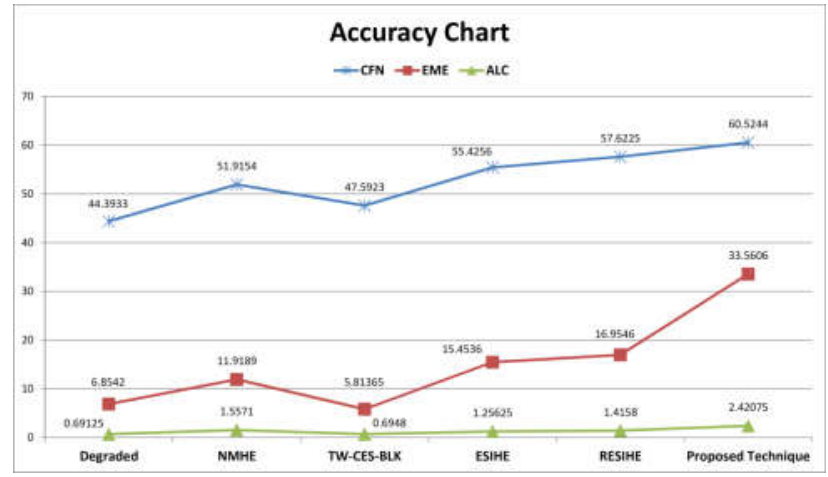

Figure 5 - Analytical graph of the average scores of Table 1.

From the comparison results shown in Fig. 3 Fig. 5 and Table 1, it can be seen that the comparative techniques performed differently, which can be justified in accordance with the variation in the nature of the used images. Regarding the NMHE technique, it gave a moderate 
performance as the processed images have minor enhancement when compared to the degraded images. In the resulting images, the brightness is increased in certain regions, the contrast is slightly improved and the colors are marginally enhanced.

Regarding the TW-CES-BLK technique, it gave a low performance as the resulting images appeared somewhat different than the degraded images. Accordingly, the brightness increased globally, the contrast is amended and the colors are slightly enhanced. In addition, this technique improved the sharpness of the resulting images, yet introduced the blocking effect, which is considered undesirable in many image processing applications. Regarding the ESIHE and RESIHE techniques, they gave relatively similar performances, slightly in favor of the RESIHE technique. Accordingly, they both improved the local and global contrast, while the colors increased to an above-average level.

Regarding the proposed technique, it performed the best in terms of recorded accuracy and perceived quality, since it provided visually pleasing results with the highest IQA scores. Accordingly, it did not amplify the brightness while improving the contrast and produced ameliorated colors for the resulting images. These facts can be observed by comparing the IQA results of the degraded and the processed images, where there are noticeable differences between such results in favor of the proposed technique. However, like many available image processing techniques, the proposed technique contains one parameter (in this case $\delta$ ), whose value must be entered manually. Such practice is followed to provide the user with more control over the processing ability of the used technique. As a future work, a suitable method can be developed to calculate the value of $\delta$ automatically. Improving the contrast of color images using a low-complexity technique is a challenging task. However, such task is successfully achieved in this study by providing a new technique that can improve the contrast using simple calculations. Finally, it is expected to extend the use of this technique to other existing image processing applications.

\section{CONCLUSION}

A new adjustable contrast stretching technique is introduced in this article, in which it is developed based on the concept of linear contrast stretching to improve the contrast of color images using few calculations. Accordingly, it is tested with various real low-contrast color images, compared with four advanced contrast enhancement techniques and the quality of the obtained results is evaluated using three eminent IQA metrics. From the obtained results, it is obvious that the proposed technique provided satisfactory results, as it produced natural contrast images with no visible artifacts and outperformed the comparative techniques by scoring the highest in terms of recorded accuracy. Thus, it is confirmed that the proposed technique is well suited for contrast enhancement of color images and can be further used in many image processing applications.

\section{REFERENCES}

[1] T. Celik and T. Tjahjadi, "Automatic image equalization and contrast enhancement using gaussian mixture modeling," IEEE Transactions on Image Processing, vol. 21, no 1, pp. 145-156, 2012.

[2] S. Naik and C. Murthy, "Hue-preserving color image enhancement without gamut problem," IEEE Transactions on Image Processing, vol. 12, no. 12, pp. 1591-1598, 2003.

[3] M. Colbert, E. Reinhard and C. Hughes, "Painting in high dynamic range," Journal of Visual Communication and Image Representation, vol. 18, no. 5, pp. 387-396, 2007.

[4] S. Syrjala, "Critique on the use of the delta distribution for the analysis of trawl survey data," ICES Journal of Marine Science, vol. 57, no. 4, pp. 831-842, 2000.

[5] B. Gupta and M. Tiwari, "Minimum mean brightness error contrast enhancement of color images using adaptive gamma correction with color preserving framework," Optik International Journal for Light and Electron Optics, vol. 127, no. 4, pp. 1671-1676, 2016.

[6] C. Jung and T. Sun, "Optimized perceptual tone mapping for contrast enhancement of images," IEEE Transactions on Circuits and Systems for Video Technology, vol. 27, no. 6, pp. 1161-1170, 2017.

[7] G. Raju and M. Nair, "A fast and efficient color image enhancement method based on fuzzylogic and histogram," AEU - International Journal of Electronics and Communications, vol. 68 , no. 3, pp. 237-243, 2014.

[8] S. Kim, J. Jeon and I. Eom, "Image contrast enhancement using entropy scaling in wavelet domain," Signal Processing, vol. 127, pp. 1-11, 2016.

[9] J. Mukherjee and S. Mitra, "Enhancement of color images by scaling the DCT coefficients," IEEE Transactions on Image Processing, vol. 17, no. 10, pp. 1783-1794, 2008.

[10] S. Poddar, D. Sharma, A. Ghosh, S. Tewary, V. Karar and S. Pal, "Non-parametric modified histogram equalisation for contrast enhancement," IET Image Processing, vol. 7, no. 7, pp. 641-652, 2013. 
[11] K. Singh and R. Kapoor, "Image enhancement using exposure based sub image histogram equalization," Pattern Recognition Letters, vol. 36, pp. 10-14, 2014.

[12] K. Singh, R. Kapoor and S. Sinha, "Enhancement of low exposure images via recursive histogram equalization algorithms," Optik - International Journal for Light and Electron Optics, vol. 126, no. 20, pp. 26192625, 2015.

[13] Z. Wang and A. Bovik, "Reduced- and noreference image quality assessment," IEEE Signal Processing Magazine, vol. 28, no. 6, pp. 29-40, 2011.

[14] A. Mittal, A. Moorthy and A. Bovik, "Noreference image quality assessment in the spatial domain," IEEE Transactions on Image Processing, vol. 21, no. 12, pp. 4695-4708, 2012.

[15] K. Zhan, J. Shi, J. Teng, Q. Li and M. Wang, "Feature-linking model for image enhancement," Neural Computation, vol. 28, no. 6, pp. 1072-1100, 2016.

[16] Y. Xiang, B. Zou and H. Li, "Selective color transfer with multi-source images," Pattern Recognition Letters, vol. 30, no. 7, pp. 682689, 2009.

[17] S. Again, B. Silver, and K. Panetta, "Transform coefficient histogram-based image enhancement algorithms using contrast entropy," IEEE Transactions on Image Processing, vol. 16, no. 3, pp. 741-758, 2007.

[18] R. Hummel, "Histogram modification techniques," Computer Graphics and Image Processing, vol. 4, no. 3, pp. 209-224, 1975.

[19] N. Hassan and N. Akamatsu, "A new approach for contrast enhancement using sigmoid function," The International Arab Journal of Information Technology, vol. 1, no. 2, pp. 221225, 2004.

[20] L. Voicu, H. Myler and A. Weeks, "Practical considerations on color image enhancement using homomorphic filtering," Journal of
Electronic Imaging, vol. 6, no. 1, pp. 108-114, 1997.

[21] C. Tsai, "Adaptive local power-law transformation for color image enhancement," Applied Mathematics \& Information Sciences, vol. 7, no. 5, pp. 2019-2026, 2013.

[22] C. Yang, "Image enhancement by modified contrast-stretching manipulation," Optics \& Laser Technology, vol. 38, no. 3, pp. 196-201, 2006.

[23] D. Jobson, Z. Rahman and G. Woodell, "Properties and performance of a center/surround retinex," IEEE Transactions on Image Processing, vol. 6, no. 3, pp. 451-462, 1997.

[24] T. De and B. Chatterji, "An approach to a generalised technique for image contrast enhancement using the concept of fuzzy set," Fuzzy Sets and Systems, vol. 25, no. 2, pp. 145$158,1988$.

[25] A. Draa and A. Bouaziz, "An artificial bee colony algorithm for image contrast enhancement," Swarm and Evolutionary Computation, vol. 16, pp. 69-84, 2014.

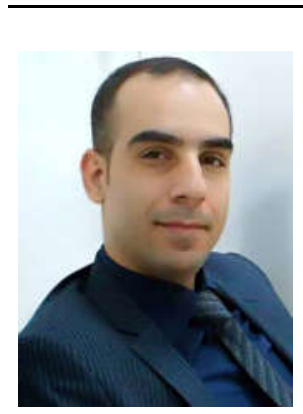

Zohair Al-Ameen was born in 1985. He received his BSc degree in Computer Science from the University of Mosul in 2008. Then, he received his $M S c$ and $P h D$ degrees in Computer Science from the Technological University of Malaysia in 2011 and 2015, respectively.

He was awarded the best student award owing to the outstanding performance in his PhD studies. His research interests include algorithms design, artificial intelligence, computer forensics, computer vision, and digital image processing. Currently, he is a full-time lecturer at the Department of Computer Science, College of Computer Science and Mathematics, University of Mosul. Finally, he has authored many articles which are published in international journals of high repute. 